Ann. Zootech., 1986, 35 (3), 205-218

\title{
Valorisation nutritive d'un grignon d'olive traité à la soude
}

\author{
J.F. AGUILERA et Eduarda MOLINA \\ avec la collaboration technique de Encarnación COLMENERO, \\ Francisca GIL et Dolores RODRIGUEZ \\ Estación Experimental del Zaidín (C.S.I.C.) \\ Profesor Albareda, 1. 18008 Granada, Espagne
}

\section{Résumé}

\begin{abstract}
Afin de mesurer l'effet du traitement à la soude sur la valeur nutritive du grignon d'olive et déterminer aussi la précision des méthodes de laboratoire pour estimer sa valeur nutritive, nous avons réalisé une série d'essais en traitant du grignon d'olive épuisé tamisé (partiellement dénoyauté) par la technique de pulvérisation avec $0,5,7,5$ et $10 \mathrm{~g}$ de $\mathrm{NaOH} / 100 \mathrm{~g}$ de sous-produit.

Le traitement à la soude diminue le contenu en $\mathrm{NDF}(\mathrm{P}<0,001)$ par suite de la solubilisation des hémicelluloses $(\mathrm{P}<0,001)$ et augmente significativement la solubilité enzymatique, la digestibilité in vitro et in vivo et donc la valeur énergétique du grignon d'olive $(\mathrm{P}<0,001)$.

Les résultats sont discutés, ainsi que la précision des différentes méthodes de laboratoire pour prédire la valeur nutritive du grignon d'olive. La méthode de mesure de la solubilité enzymatique est très convenable, et présente des avantages par rapport aux autres méthodes.

En conclusion, la valeur nutritive du grignon d'olive est faible et, malgré l'importance de l'effet du traitement à la soude, elle reste toujours inférieure à celle des pailles de céréales. Cependant, le grignon peut être utilisé en quantité modérée dans des régimes d'entretien et en quantité plus élevée dans les cas de disette.
\end{abstract}

Mots clés : Grignon d'olive, traitement à la soude, digestibilité, valeur énergétique.

\section{Introduction}

L'utilisation des grignons d'olive, le plus important des sous-produits de l'industrie oléicole, dans la nutrition animale, est très limitée du fait de leur faible valeur nutritive. Cependant, la valorisation de ce sous-produit prend une importance pratique particulière, étant donné que les grignons d’olive sont produits dans les régions où les fourrages sont limités.

La presque totalité de la production mondiale d'olives, évaluée aux environs de 8400000 T (MAPA, 1982), provient des pays du Bassin Méditerranéen. Quatre pays (Italie, Grèce, Espagne et Tunisie) fournissent 74 p. 100 de cette quantité. Les 
grignons d'olive sont le principal résidu de l'extraction de l'huile et sa production totale est estimée à $3000000 \mathrm{~T}$ par an dont $480000 \mathrm{~T}$ pour l'Espagne.

Les données existantes sur la digestibilité des grignons d'olive sont rares et extraordinairement variables en fonction de son origine, de la technologie appliquée pendant l'extraction de l'huile et de la méthode de conservation. Mais, en tout cas, ils reflètent une faible utilisation digestive. Les valeurs les plus faibles correspondent aux grignons bruts et les plus élevées aux pulpes (BozA \& VARELA, 1961 ; BozA et al., 1970 ; Duranti et al., 1978 ; Martilloti, 1984 ; Nefzaoui, 1985).

Etant donnée la composition chimique des grignons d'olive, dont les parois cellulaires sont très lignifiées, nous avons estimé que le traitement à la soude suivi d'ensilage était le procédé le plus approprié pour améliorer leur valeur nutritive (NeFZAOUI, 1985).

D’un autre côté, il est nécessaire de développer l'application de méthodes de laboratoire permettant de prédire avec précision la valeur nutritive de ces sousproduits et facilitant l'inclusion des grignons d'olive dans des rations pratiques.

C'est pourquoi nous nous proposons :

a) d'étudier l'effet du traitement à la soude des grignons épuisés tamisés sur leur composition chimique et leur digestibilité in vivo;

b) de connaître la précision de deux méthodes de laboratoire (digestibilité in vitro et solubilité enzymatique) pour estimer la valeur nutritive de ces grignons traités à la soude.

\section{Matériel et méthodes}

\section{A. Grignons}

Nous avons utilisé du grignon d'olive épuisé tamisé (Nomenclature adoptée dans le Séminaire International sur la Valorisation des Sous-produits de l'Olivier organisé par la FAO, Madrid 1983, pour «le résidu obtenu au niveau industriel après épuisement et élimination partielle, par procédé mécanique, des débris de noyaux »). La composition du grignon, en pourcentage de la matière sèche, est la suivante : matières grasses 2,7; MAT 10,6; cellulose brute 33,4; NDF 62,9; ADF 52,9; ADL 27,4 ; cendres 7,7. Le sous-produit a été traité avec des solutions de $\mathrm{NaOH}$ selon la technique de pulvérisation. Les doses de soude appliquées étaient : $0,5,7,5$ et $10 \mathrm{~g} / 100 \mathrm{~g}$ de grignon (150 kg de sous-produit pour chacune des doses). Le matériel ainsi traité a été conservé pendant 3 semaines à la température ambiante, dans des récipients fermés.

\section{B. Schéma expérimental}

On a déterminé l'effet du traitement à la soude sur la composition chimique, sur la solubilité enzymatique et sur la digestibilité in vitro et in vivo du grignon d'olive. Les techniques analytiques utilisées ont été déjà décrites (Molina et al., 1983). Pour le dosage de l'énergie brute des aliments et des fèces nous avons utilisé une bombe calorimétrique adiabatique. 
La digestibilité in vitro a été déterminée selon la technique de Tilley \& TerRY (1963). Le jus de rumen provenait de moutons de race Segureña nourris à base de foin de luzerne et $150 \mathrm{~g}$ de soja par jour. La solubilité enzymatique a été déterminée selon la technique d'AUFRERE (1982). La préparation cellulasique employée est extraite de Trichoderma viride (BHD N. 39074).

Les essais in vivo ont été conduits d'après le schéma suivant :

\section{Animaux}

Nous avons utilisé 12 moutons de race Segureña, mâles castrés (âgés de 12 mois et d'un poids de $40 \pm 0,75 \mathrm{~kg}$ au début des essais), regroupés selon leur poids en deux groupes de 6 animaux. Chaque groupe a été divisé en 2 lots de 3 animaux. Ces moutons étaient placés dans des cages à métabolisme. Après une période d'adaptation de 3 semaines au régime correspondant additionné de $20 \mathrm{~g}$ d'un composé minéral et vitaminé, on a mesuré exactement les quantités excrétées pendant 10 jours. L'eau était distribuée à volonté.

\section{Régimes}

Nous avons constitué 4 régimes témoins formés par $400 \mathrm{~g}$ de foin de luzerne, de $500 \mathrm{~g}$ de concentré (orge + tourteau de tournesol) et de $100 \mathrm{~g}$ de grignon, non traité ou traité selon les régimes (tabl. 1). En effet, nous avons pensé que la présence du grignon dans les régimes témoins éviterait des phénomènes associatifs, difficiles à contrôler, qui pourraient apparaître avec l'incorporation du grignon dans les régimes expérimentaux. Les phénomènes associatifs peuvent induire des résultats erronés de digestibilité du grignon, calculée par différence.

Les régimes expérimentaux ont été formulés en remplaçant $200 \mathrm{~g}$ de la quantité du régime témoin offerte aux animaux par jour par $350 \mathrm{~g}$ de grignon d'olive traité avec la dose de soude correspondante. On a considéré que les quantités remplacées étaient à peu près isocaloriques.

\section{Déroulement des essais}

Au cours de deux essais successifs selon le schéma du tableau 2, les animaux ont reçu les régimes témoins et les régimes expérimentaux correspondants afin de calculer, par différence, la digestibilité et la valeur énergétique du grignon d'olive. Le niveau d'ingestion était proche de celui de l'entretien (700 et $850 \mathrm{~g} /$ jour respectivement pour les régimes témoins et expérimentaux).

Afin de déterminer la rétention d'énergie due à l'ingestion de grignon, nous avons effectué une série de mesures de la production de $\mathrm{CH}_{4}$ et $\mathrm{CO}_{2}$ ainsi que de la consommation d' $\mathrm{d}_{2}$ pendant 24 heures, dans des chambres respiratoires à circuit ouvert (Aguilera \& Prieto, 1985), avec des animaux qui recevaient $700 \mathrm{~g}$ du régime témoin et ce même régime additionné de $350 \mathrm{~g}$ de matière sèche du grignon traité. Ces mesures ont été faites à la fin des essais de digestibilité dans le cas des régimes témoins, et après une période d'adaptation de 3 semaines dans le cas des régimes témoins additionnés de $350 \mathrm{~g}$ du grignon traité. 


\section{Tableau 1}

Composition des régimes témoins et expérimentaux.

Chemical composition of control and experimental diets.

\begin{tabular}{|c|c|c|c|c|c|c|c|c|}
\hline $\begin{array}{l}\text { Régimes } \\
\text { Diets }\end{array}$ & $\mathrm{RT}_{0}$ & $\mathrm{RT}_{5}$ & $\mathrm{RT}_{7.5}$ & $\mathrm{RT}_{10}$ & $\mathrm{RE}_{0}$ & $\mathrm{RE}_{\tilde{5}}$ & $\mathrm{RE}_{\overline{7}, 5}$ & $\mathrm{RE}_{10}$ \\
\hline \multicolumn{9}{|l|}{$\begin{array}{l}\text { Composition alimentaire, } \mathrm{g} / \mathrm{kg} \\
\text { Feed composition, } \mathrm{g} / \mathrm{kg}\end{array}$} \\
\hline $\begin{array}{l}\text { Foin de luzerne } \ldots \ldots \ldots \ldots \\
\text { Lucerne hay }\end{array}$ & 400 & 400 & 400 & 400 & 235 & 235 & 235 & 235 \\
\hline $\begin{array}{l}\text { Grignon traité avec } 0 \% \mathrm{NaOH} \\
\text { Olive cake } \\
\text { treated with } 0 \% \mathrm{NaOH}\end{array}$ & 100 & - & - & - & 471 & - & - & 一 \\
\hline $\begin{array}{l}\text { Grignon traité avec } 5 \% \mathrm{NaOH} \\
\text { Olive cake } \\
\text { treated with } 7 \% \mathrm{NaOH}\end{array}$ & - & 100 & 一 & - & - & 471 & - & 一 \\
\hline $\begin{array}{l}\text { Grignon traité avec } 7,5 \% \\
\mathrm{NaOH} \ldots \ldots \ldots \ldots \ldots \ldots \ldots \ldots \\
\text { Olive cake } \\
\text { treated with } 5 \mathrm{NaOH}\end{array}$ & 一 & - & 100 & - & - & - & 471 & $\rightarrow$ \\
\hline $\begin{array}{l}\text { Grignon traité avec } 10 \% \\
\mathrm{NaOH} \ldots \ldots \ldots \ldots \ldots \ldots \ldots \\
\begin{array}{l}\text { Olive cake } \\
\text { treated with } 10 \%\end{array}\end{array}$ & - & 一 & 一 & 100 & 一 & - & - & 471 \\
\hline Orge - Barley ........... & 340 & 340 & 340 & 340 & 200 & 200 & 200 & 200 \\
\hline $\begin{array}{l}\text { Tourteau de tournesol } \ldots \ldots \\
\text { Sunflower meal }\end{array}$ & 160 & 160 & 160 & 160 & 94 & 94 & 94 & 94 \\
\hline $\begin{array}{l}\text { Composition chimique, \% MS } \\
\text { Chemical composition, \% DM }\end{array}$ & & & & & & & & \\
\hline $\begin{array}{l}\text { MAT } \ldots \ldots \ldots \ldots \ldots \ldots \\
\text { Crude protein }\end{array}$ & 13,3 & 13,0 & 12,8 & 12,8 & 12,4 & 12,0 & 11,7 & 11,7 \\
\hline 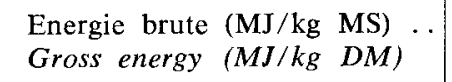 & 19,2 & 18,8 & 18,9 & 18,9 & 19,9 & 18,7 & 19,2 & 19,0 \\
\hline $\begin{array}{l}\text { Matière organique } \ldots \ldots \ldots \\
\text { Organic matter }\end{array}$ & 92,6 & 90,2 & 89,6 & 88,5 & 92,7 & 88,9 & 87,9 & 86,1 \\
\hline $\begin{array}{l}\text { Matière sèche }(\%) \ldots \ldots \ldots \ldots \\
\text { Dry matter }(\%)\end{array}$ & 88,2 & 87,6 & 87,4 & 87,8 & 79,6 & 76,2 & 75,3 & 77,2 \\
\hline
\end{tabular}

C. Analyses statistiques

Les résultats expérimentaux ont été soumis à une analyse de variance et les différences entre traitements ont été testées par la méthode de la plus petite différence significative de Duncan. 


\section{TABLEAU 2}

Schéma expérimental des mesures

sur la valeur nutritive d'un grignon d'olive traité à la soude (1).

Experimental design used to determine the feeding value of a $\mathrm{NaOH}$ treated olive cake.

\begin{tabular}{|c|c|c|c|c|c|}
\hline & & \multicolumn{2}{|c|}{$\begin{array}{c}\text { Groupe } 1 \\
\text { Group } 1\end{array}$} & \multicolumn{2}{|c|}{$\begin{array}{c}\text { Groupe } 2 \\
\text { Group } 2\end{array}$} \\
\hline & & Lot A & Lot B & Lot $\mathrm{C}$ & Lot D \\
\hline Moutons/lot & Sheep/lot $\ldots . .$. & 3 & 3 & 3 & 3 \\
\hline \multirow{2}{*}{$\begin{array}{l}\text { Essai } 1 \\
\text { Experiment } 1\end{array}$} & $\begin{array}{l}\text { Période } 1 \\
\text { Period } 1\end{array}$ & $\mathrm{RT}_{0}$ & $\mathrm{RE}_{0}$ & $\mathrm{RT}_{7,5}$ & $\mathrm{RE}_{7,5}$ \\
\hline & $\begin{array}{l}\text { Période } 2 \quad \ldots \ldots \\
\text { Period } 2\end{array}$ & $\mathrm{RE}_{0}$ & $\mathrm{RT}_{0}$ & $\mathrm{RE}_{7,5}$ & $\mathrm{RT}_{7, \mathbf{5}}$ \\
\hline \multirow{2}{*}{$\begin{array}{l}\text { Essai } 2 \\
\text { Experiment } 2\end{array}$} & $\begin{array}{l}\text { Période } 1 \\
\text { Period } 1\end{array}$ & $\mathrm{RT}_{5}$ & $\mathrm{RE}_{5}$ & $\mathrm{RT}_{10}$ & $\mathrm{RE}_{10}$ \\
\hline & $\begin{array}{l}\text { Période } 2 \\
\text { Period } 2\end{array}$ & $\mathrm{RE}_{5}$ & $\mathrm{RT}_{5}$ & $\mathrm{RE}_{10}$ & $\mathrm{RT}_{10}$ \\
\hline
\end{tabular}

(1) $\mathrm{RT}=$ Régime témoin ; $\mathrm{RE}=$ Régime expérimental. Le suffixe indique le pourcentage de soude dans le grignon d'olive de la ration.

$R T=$ Control diet $; R E=$ Experimental diet. Subscripts indicate the $\mathrm{NaOH}$ percentage in the dietary cake.

\section{Résultats}

\section{A. Composition chimique}

La conservation du grignon d'olive traité dans des conditions anaérobies est bonne et nous avons observé, malgré un $\mathrm{pH}$ élevé, une certaine fermentation.

Les résultats de l'effet du traitement à la soude du grignon d'olive sur la composition chimique sont rapportés dans le tableau 3. Dans le but d'éliminer l'effet de la variation du contenu en cendres provoquée par l'addition de la soude nous avons calculé les résultats par rapport à la matière organique.

La fraction NDF représente dans le grignon non traité 68 p. 100 de la matière organique et les composants lignocellulosiques (ADF) 84 p. 100 de la fraction NDF. Le pourcentage de cellulose est à peu près celui de la lignine.

Le traitement du grignon à la soude diminue significativement $(P<0,001)$ la fraction pariétale (NDF). Cette diminution est due à la solubilisation des hemicelluloses $(P<0,001)$. Les contenus en $\mathrm{ADF}$ et $\mathrm{ADL}$ ne changent pas, du point de vue statistique, à la suite du traitement à la soude.

La teneur en azote du grignon d'olive est faible. Cet azote est attaché, dans sa plus grande partie $(75$ p. 100$)$, aux parois cellulaires. Le traitement à la soude ne solubilise pas l'azote attaché à ces parois. 


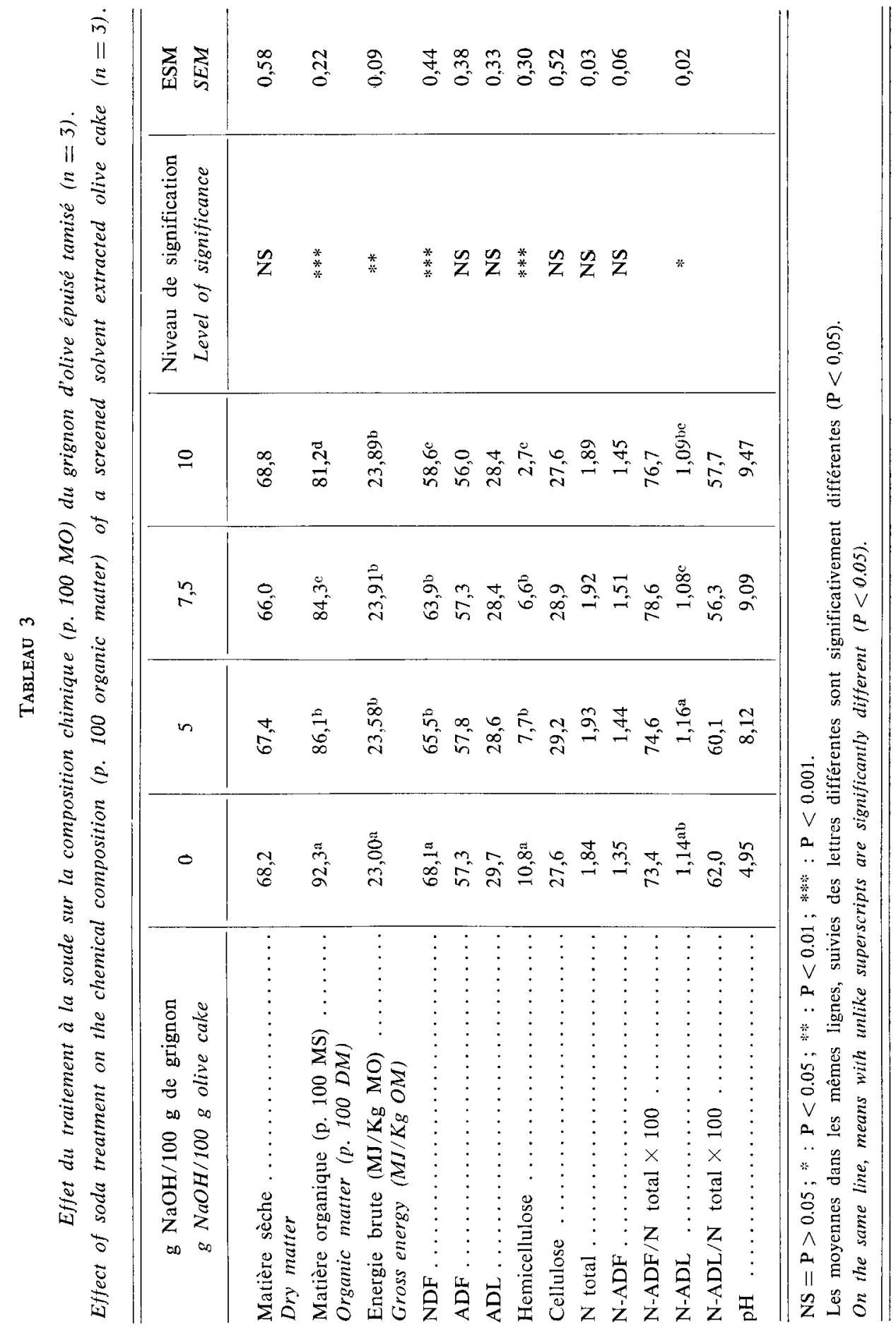




\section{B. Digestibilité in vivo}

Les résultats des essais in vivo sont présentés dans le tableau 4.

Nous n’avons pas trouvé de différences significatives entre les régimes témoins en ce qui concerne la digestibilité de la matière sèche, matière organique et énergie ainsi que pour l'utilisation digestive de l'azote.

Les mesures de bilan énergétique réalisées dans des chambres respiratoires ont montré que le niveau alimentaire des régimes témoins était légèrement supérieur à celui de l'entretien. En effet, les ingestions d'énergie métabolisable avec les régimes témoins qui contenaient du grignon traité avec $0,5,7,5$ et $10 \mathrm{~g} \mathrm{NaOH} / 100 \mathrm{~g}$ de sous-produit, étaient, respectivement : $6761( \pm 149), 7027( \pm 66), 6879$ (士50) et 7155 ( \pm 113 ) $\mathrm{KJ} /$ jour et les rétentions énergétiques correspondantes de 610 ( \pm 418$), 951$ ( \pm 334$)$. $768( \pm 135)$ et $935( \pm 381) \mathrm{KJ} /$ jour.

La substitution de $200 \mathrm{~g}$ du régime témoin par $350 \mathrm{~g}$ de grignon traité avec la dose de soude correspondante provoque des diminutions de l'utilisation digestive de la matière sèche, de la matière organique, des MAT et de l'énergie. La digestibilité de la matière sèche des régimes expérimentaux s'améliore significativement $(P<0,001)$ avec le traitement à la soude du grignon, mais l'augmentation est seulement de 4 points maximum. Par contre, ni la digestibilité de la matière organique ni celle des MAT des régimes expérimentaux sont significativement affectées par le traitement à la soude du grignon d'olive. La digestibilité de l'énergie augmente significativement $(P<0,001)$ quand on ajoute des doses de soude supérieures à $5 \mathrm{~g} / 100 \mathrm{~g}$ de grignon. Le contenu en énergie métabolisable par rapport à celui de l'énergie brute (EM/EB, p. 100) s'améliore significativement $(P<0,001)$ avec les doses croissantes de soude. Le rapport $\mathrm{EM} / \mathrm{ED}(\mathrm{p} .100)$ augmente $(\mathrm{P}<0,01)$ avec l'addition de soude mais on n'a pas remarqué de différences significatives pour les diverses doses d'alcali.

Les valeurs de digestibilité du grignon traité à la soude ont été obtenues, par différence, à partir des résultats correspondants des régimes témoins et expérimentau. On peut remarquer que les digestibilités de la matière sèche et de la matière organique sont significativement $(\mathrm{P}<0,001$ et $\mathrm{P}<0,01$ respectivement) améliorées par le traitement à la soude. Cet effet significatif du traitement à la soude sur la digestibilité in vivo a été corroboré par les résultats que nous avons obtenus in vitro. Ces valeurs reflètent une faible utilisation nutritive du grignon d'olive. La digestibilité de l'énergie ainsi que le rapport énergie métabolisable/énergie brute (p. 100) du grignon s'améliorent significativement $(\mathrm{P}<0,001)$ avec le traitement à la soude $(13,7$ et 13,4 points respectivement). Par conséquent, les teneurs en ED et en EM varient de 5,41 et 4,22 $\mathrm{MJ} / \mathrm{Kg}$ MS jusqu'à 7,61 et 6,46 MJ/Kg MS respectivement.

Nous avons obtenu des équations de régression linéaires (tabl. 5) à partir des données de digestibilité in vivo de la matière sèche, la matière organique et l'énergie et des données correspondant à la composition chimique et à la dégradabilité, qui permettent d'estimer avec une précision raisonnable, la digestibilité et la valeur énergétique du grignon testé.

La production de $\mathrm{CH}_{4}$ attribuable à l'ingestion de grignon d'olive, calculée par différence, a été de $9,3( \pm 0,62), 10,3( \pm 0,39), 9,8( \pm 0,53)$ et de $11,2( \pm 0,99)$ litres $/ \mathrm{Kg}$ MO respectivement pour le grignon traité avec $0,5,7,5$ et $10 \mathrm{~g} \mathrm{NaOH} / 100 \mathrm{~g}$ de sous-produit. Ces productions ne sont pas statistiquement différentes. 


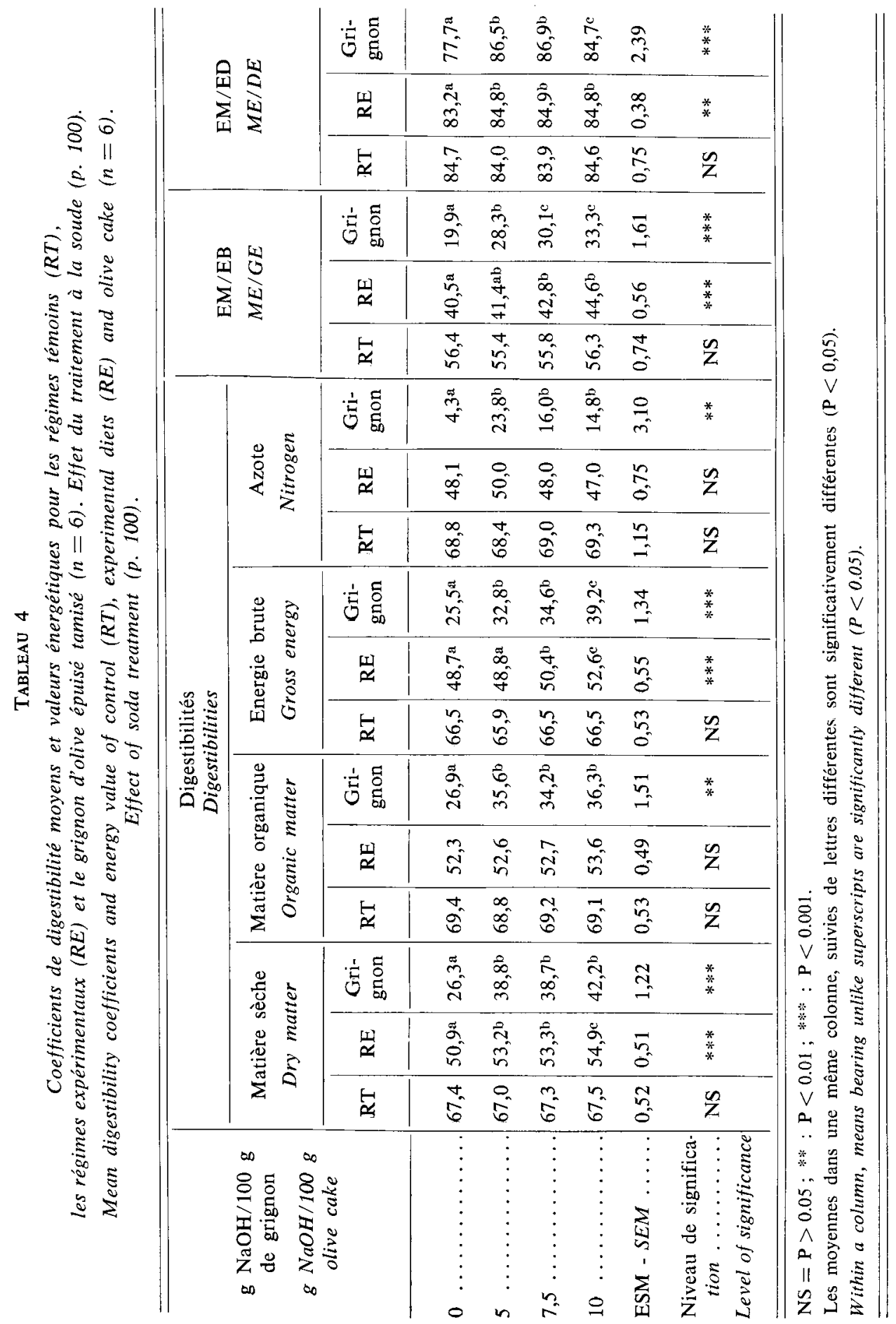


L'addition de soude augmente la digestibilité des MAT $(\mathrm{P}<0,01)$ du grignon mais l'azote utilisable est très réduit.

\section{Discussion-conclusions}

Il est très difficile de comparer nos résultats de composition chimique du grignon d'olive avec les données bibliographiques car la technologie d'extraction de l'huile et son efficacité modifient les proportions relatives des constituants des grignons qui montrent, par conséquent, une composition chimique très variable.

Les données correspondant à la composition chimique du grignon d'olive sont voisines de celles acquises avec des grignons épuisés et partiellement tamisés (SANSOUCY, 1984 ; NefzaOur, 1985). Le grignon d'olive est un matériel très fibreux et à faible contenu en azote, fraction qui est attachée à la paroi cellulaire, très lignifiée. Les composants des parois cellulaires du grignon constituent un complexe peu accessible aux enzymes bactériennes du rumen. De même qu'avec d'autres sous-produits lignocellulosiques, la soude agit sur ce complexe-là en modifiant sa structure. Cette modification se traduit par une diminution de la fraction NDF due, surtout, à la solubilisation des hémicelluloses (JACKSON, 1977 ; Molina et al., 1983). Nos résultats sont comparables à ceux obtenus avec des grignons par Molina et al. (1984) et NeFZAOUI (1985).

Le faible contenu en azote, lié dans sa plus grande partie aux parois cellulaires et le fait qu'il n'est pas solubilisé par l'addition de soude sont les causes de sa faible disponibilité (Yu et al., 1975 ; VAN SoEST, 1982). Pour obtenir une dégradation optimale, dans le rumen, de la matière organique du grignon, il faudra donc complémenter ce sous-produit (Molina et al., 1984 ; NefZaOui, 1985) en MAT.

Les résultats de mesures in vivo réalisées avec des grignons d'olive sont très rares. Par ailleurs, la composition de la ration, le niveau d'alimentation et la méthode choisie pour estimer la digestibilité peuvent modifier les résultats de la digestibilité de ce sous-produit.

Nous avons observé dans nos essais des augmentations de la digestibilité de la matière sèche, de la matière organique et de l'énergie du grignon d'olive après traitement à la soude. L'augmentation est du même ordre de grandeur que celles obtenues par FeIst et al. (1970), Guggolz et al. (1971) et Choung \& McManus (1976) avec des fourrages très lignifiés et par Nefzaour (1985) avec des grignons et des pulpes d'olive. Bien que l'amélioration de la valeur nutritive du grignon d'olive par la soude soit importante, le sous-produit a toujours une valeur nutritive inférieure à celle des pailles de céréales traitées à la soude (VERma \& JACKson, 1975 ; Dulphy et al., 1982 ; Dulphy et al., 1983). L'intensité de l'effet du traitement à la soude augmente peu au fur et à mesure que la dose d'alcali augmente. Il est possible que cela soit explicable par l'augmentation de la vitesse de passage à travers le tractus digestif, causée par une plus grande consommation d'eau avec la soude (BolduAN et al., 1974 ; McManus et al., 1976 ; Berger et al., 1980). Ololade et al. (1972) considèrent aussi que l'augmentation de la pression osmotique dans le rumen due à la présence de $\mathrm{Na}$ contribue à l'absence d'augmentation de l'effet du traitement à la soude avec les doses élevées. De même, cette présence peut réduire l'activité microbienne ruminale (BERGEN, 
1970). Cependant, nous n'avons pas observé, pour la production de $\mathrm{CH}_{4}$ attribuée à la fermentation du grignon, de différences dépendant du niveau de soude ajoutée. Dans tous les cas, la production de $\mathrm{CH}_{4}$ était modérée et elle présentait une tendance à augmenter avec le traitement à la soude. NeFZAOU et al. (1983) ont observé que l'ingestion des grignons d'olive induisait une faible production d'acides gras volatils dans le rumen (51-65 $\mathrm{mMol} / \mathrm{l})$ dont la proportion correspondait aux fermentations caractéristiques des aliments fibreux. Le traitement à la soude (4 p. 100) augmentait légèrement la teneur d'acides gras volatils dans le rumen (71-73 mMol/1) sans modifier leurs proportions relatives. DulPHY et al. (1983), avec des pailles de céréales traitées aux alcalis, ont obtenu des résultats très similaires.

L'utilisation digestive de l'azote du grignon est toujours faible parce qu'il est attaché (75 p. 100) aux parois cellulaires et il n'est pas solubilisé par le traitement à la soude.

Nous n'avons pas trouvé dans la bibliographie des équations spécifiques nous permettant de prédire la valeur nutritive des grignons d'olive. Etant donnée la composition $\mathrm{du}$ sous-produit (très lignifié), le calcul des valeurs énergétiques à partir des équations basées sur les contenus des nutriments bruts déterminés par l'analyse classique est soumis à des erreurs très importantes. Par exemple, à partir des données de digestibilité in vitro de la matière organique des grignons d'olive procurés par l'ADAS (Agriculture Development and Advisory Service) en Grande-Bretagne, on a obtenu une valeur de $4,1 \mathrm{MJ} / \mathrm{Kg}$ MS pour le contenu en EM de ces sous-produits (Morgan \& Trinder, 1980). Ce chiffre a remplacé celui de 12,7 MJ/Kg MS calculé par rapport aux teneurs des nutriments bruts (MAFF, 1975). La valeur de 4,1 est proche de $4,22 \mathrm{MJ} / \mathrm{Kg} \mathrm{MS}$, provenant de nos essais in vivo pour l'EM du grignon d'olive non traité.

Nos résultats indiquent que la solubilité enzymatique est la méthode la plus précise pour estimer la valeur nutritive du grignon traité par rapport à celle de la digestibilité in vitro. De plus, les techniques enzymatiques sont très simples. DemarQUILLY \& JARRIGE (1981) ainsi que MCLEOD \& Minson (1978) considèrent que les méthodes de solubilité enzymatique ont une précision équivalente à celle des méthodes de digestibilité in vitro pour estimer la digestibilité in vivo des aliments des ruminants.

On peut conclure que, même si le traitement à la soude du grignon d'olive épuisé tamisé augmente sa valeur nutritive, celle-ci est faible et reste généralement inférieure à celle des pailles de céréales non traitées (5,6-7,3 MJ EM/Kg MS ; MAFF, 1975). Il faudra donc introduire ce sous-produit en quantités modérées dans les régimes d'entretien. On pourra l'introduire en proportion plus élevée dans des situations de disette seulement.

\section{Remerciements}

Nous voudrions présenter nos sincères remerciements à l'entreprise EsPUNY e Hijos, S.A. qui nous a procuré le grignon d'olive et à M. LARA qui a collaboré au traitement des analyses statistiques. 


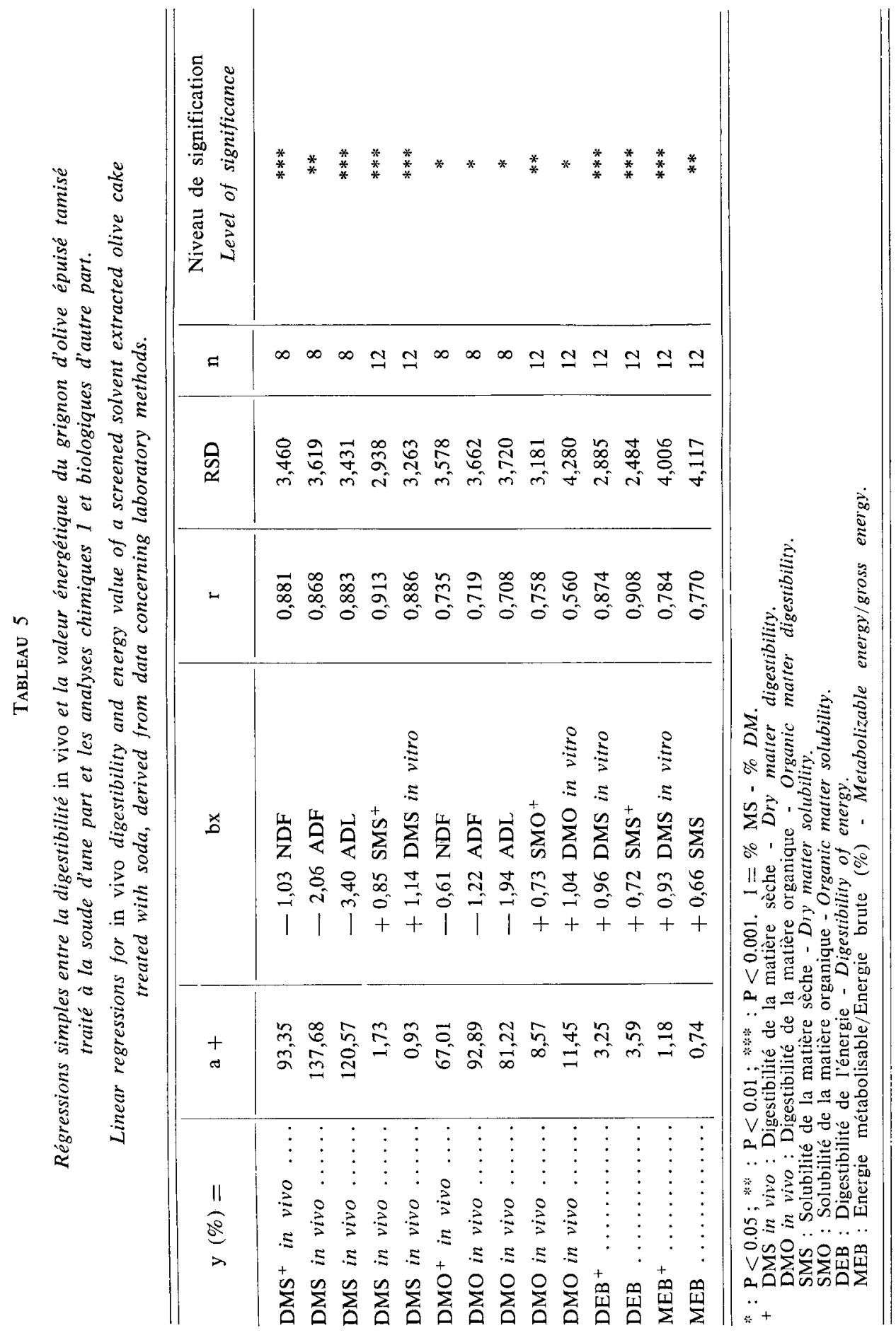




\section{Summary \\ Effect of soda treatment on the feeding value of olive cakes}

The effect of soda treatment on the feeding value of olive cakes and the accuracy of laboratory methods as predictors of its digestibility and energy content were studied. A screened and extracted olive cake was sprayed with $0,5,7.5$ and $10 \mathrm{~g} \mathrm{NaOH} / 100 \mathrm{~g}$ by-product.

Soda treatment significantly reduced NDF $(P<0.001)$, due to solubilisation of hemicelluloses ( $P<0.001)$ (table 3 ), and significantly improved the solubility in cellulase and the in vitro and in vivo digestibilities. Thus, the energy value of the olive cake was increased $(\mathrm{P}<0.001$ ) (table 4).

The accuracy of the different Iaboratory methods for estimating the nutritive value of olive cake is discussed (table 5). Degradability in cellulase from Trichoderma viride seems to be a very suitable method for estimating in vivo digestibility and to present advantages as compared to other methods.

In conclusion, in spite of the improvement produced by the soda treatment, the feeding value of the olive cake remains lower than that of non treated cereal straws. It is recommended to use it in moderate amounts in maintenance diets and in higher amounts only in case of feed shortage.

Key words : Olive cake, soda treatment, digestibility, energy value.

Reçu en décembre 1985.

Accepté en mai 1986.

\section{Références bibliographiques}

Agricultural Research Council, 1980. The Nutrient Requirements of Ruminant Livestock. Commonwealth Agricultural Bureaux. Londres, $351 \mathrm{p}$.

Aguilera J.F., Prieto C., 1985. An open-circuit respiration plant for animals of different sizes In : Proc. 10th International Symp. on Energy Metabolism. Airlie, September 15-21, pp. 159-162.

Aufrere J., 1982. Etude de la prévision de la digestibilité des fourrages par une méthode enzymatique. Ann. Zootech., 31, 111-130.

Bergen W.G., 1970. Osmolality and rumen function in sheep. J. Anim. Sci., 31, 236 (Abstr.).

Berger L.L., Klopfenstein T.J., Britton R.A., 1980. Effect of sodium hydroxide treatment on rate of passage and rate of ruminal fiber digestion. J. Anim. Sci., 50, 745-749.

Bol.duan G., Voigt J., Piatkowski B., 1974. Untersuchungen zum Aufschluss von Getreidestroh. 3. Einfluss der Behandlung mit Natronlauge auf die Pansenfermentation in Versuchen and Kuhen. Arch. Tierernähr., 24, 149-157.

Boza J., Varela G., 1961. Digestibilidad y valor nutritivo en cerdos ibéricos del orujo bruto de aceitunas. Granja, 9, 31-34 et 49-51.

Boza J., Fonolla J., Aguilera J.F., 1970. Aprovechamiento de subproductos agrícolas industriales en la alimentación del ganado ovino. 1. Estudio de la digestibilidad de dietas a base de orujo de aceituna y melazas de remolacha. Rev. Nutr. Animal, 8, 13-22.

Choung C.C., McManus W.R., 1976. Studies on forage cell walls. 3. Effect of feeding alkalitreated rice hulls to sheep. J. Agric. Sci. Camb., 86, 517-530.

Demarquilly C., Jarrige R., 1981. Panorama des méthodes de prévision de la digestibilité et de la valeur énergétique des fourrages. I.N.R.A., pp. 41-59.

Dulphy J.P., Breton J., Bienaime A., Louyot J.M., 1982. Etude de la valeur alimentaire des pailles de céréales traitées ou non à la soude. I. Influence du traitement à la soude. Ann. Zootech., 31, 195-214. 
Dulphy J.P., Breton J., LouYot J.M., Bienaime A., 1983. Etude de la valeur alimentaire des pailles de céréales traitées ou non à la soude. III. Influence du niveau d'apport d'aliment concentré. Ann. Zootech., 32, 53-80.

Duranti E., Polidori P., Rongoni V., Sarti D.M., 1978. Composizione chimica, digeribilitá, valore nutritivo di una sausa vergine derivata da pasta snocciolata di olive addizionate di enzimi. Ann. Fac. Agr. Univ. Perugia, 32, 413-431.

Feist W.C., Baker A.J., Takkow H., 1970. Alkali requirements for improving the digestibility of hardwoods by rumen micro-organisms. J. Anim. Sci., 30, 832-835.

Guggolz J., McDonald G.M., Walter H.G., Jr., Garret W.N., Kohler G.O., 1971. Treatment of farm wastes for livestock feed. J. Anim. Sci., 33, 284 (Abstr.).

JACKson M.G., 1977. Review article - the alkali treatment of straws. Anim. Feed Sci. Technol., 2, 105-130.

MAFF, 1975. Energy allowances and feeding systems for ruminants. Technical Bulletin, 33, HMSO. Londres, $79 \mathrm{p}$.

MAPA, Ministerio de Agricultura, Pesca y Alimentacion, 1982. Annuario de Estadística Agraria. Madrid, $682 \mathrm{p}$.

Martilloti F., 1984. Utilisation des sous-produits de l'olive dans l'alimentation animale en Italie. In : Valorisation des Sous-Produits de l'Olivier. Réunion du groupe de travail organisée par le Projet Régional D'amélioration de la Production Oleicole. FAO, Madrid, 17-18 novembre 1983 , pp. 48-58.

MCLeod M.N., Minson D.J., 1978. The accuracy of the pepsin-cellulase technique for estimating the dry matter digestibility in vivo of grasses and legumes. Anim. Feed Sci. Technol., 3, 277-287.

McManus W.R., Choung C.C., Robinson V.N.E., 1976. Studies of forage cell walls. Flow and degradation of alkali-treated rice hull digesta in the ruminant digestive tract. J. Agric. Sci. Camb., 87, 471-483.

Molina E., Boza J., Aguilera J.F., 1983. Nutritive value for ruminants of sugar cane bagasse ensiled after spray treatments with different levels of $\mathrm{NaOH}$. Anim. Feed Sci. Technol., 9, 1-17.

Molina E., Agullera J.F., Boza J., 1984. Traitement à l'alcali du grignon d'olive tamisé épuisé. Effet sur la composition chimique et la digestibilité «in vitro». In : Valorisation des Sous-Produits de l'Olivier. Réunion du groupe de travail organisée par le Projet Régional D'amélioration de la Production Oleicole. FAO, Madrid, 17-18 novembre 1983, pp. 117-121.

Morgan D.E., Trinder H., 1980. The composition and nutritional value of some tropical and sub-tropical by-products. In : By-products and Wastes in Animal Feeding. British Society of Animal Production. Occasional Publication No 3, 91-111.

NefZaOu A., 1985. Valorisation des résidus lignocellulosiques dans l'alimentation des ruminants par les traitements aux alcalis. Application aux grignons d'olive. Thèse Docteur Ing. Faculté des Sciences. Université Catholique de Louvain, 345 p.

Nefzaoui A., Helling Ph., Vanbelle M., 1983. Ensiling olive pulp with ammonia : Effects on voluntary intake and digestibility measured by sheep. 34th Annual Meeting of the study commission E.A.A.P. Madrid, 3-6 octobre, p. 198.

Ololade B.G., Mowat D.N., Yao Y.T., Sмith G.C., 1972. Sodium hydroxide - treated diet and body fluids parameters. J. Anim. Sci., 35, 232 (Abstr.).

SANSOUCY R., 1984. Utilisation des sous-produits de l'olivier en alimentation animale dans le bassin méditerranéen. In : Valorisation des Sous-Produits de l'Olivier. Réunion du groupe de travail organisée par le Projet Régional D'amélioration de la Production Oleicole. FAO, Madrid, 17-18 novembre 1983, pp. 65-107. 
Tilley J.M.A., Terry R.A., 1963. A two stage technique for the in vitro digestion of forage crops. J. Br. Grassl. Soc., 18, 104-111.

Van Soest P.J., 1982. Nutritional Ecology of the Ruminant. (Ed. Van Soest P.J.) O \& Books, Inc., Oregon, 374 p.

Verma M.L., JACKSON M.G., 1975. The comparativeness of sodium hydroxide and calcium oxide in increasing the in vitro, nylon-bag digestibility of various roughages. In : Improved utilization of agricultural waste materials and industrial by-products as livestock feed. Research Progress Report 1969-1974. G.B. Pant University, Pantnagar, pp. 14-15.

Yu Y., Thomas J.W., Emery R.S., 1975. Estimated nutritive value of treated forages for ruminants. J. Anim. Sci., 41, 1742-1751. 